\title{
Pasif Başkalaşımın Çıkış Varyansı Kısıtlı Kontrolcü İle Yönlendirilen İha Üzerinde Etkileri
}

\author{
Firat Şal ${ }^{1 *}$ \\ 1 İskenderun Teknik Üniversitesi, Havacıllk ve Uzay Bilimleri Fakültesi Fakültesi, Uçak Gövde-Motor Bölümü, Hatay, Türkiye (ORCID: 0000-0003-2412-4131)
}

(Ilk Geliş Tarihi 20 Ocak 2020 ve Kabul Tarihi 23 Şubat 2020)

(DOI: 10.31590/ejosat.682796)

ATIF/REFERENCE: Şal, F. (2020). Pasif Başkalaşımın Çıkış Varyansı Kısıtlı Kontrolcü İle Yönlendirilen İha Üzerinde Etkileri. Avrupa Bilim ve Teknoloji Dergisi, (18), 239-242.

\section{$\ddot{O} \mathbf{z}$}

Bu çalışma çıkış varyansı kısıtlı kontrolcü (OVC) ile yönlendirilen pasif başkalaşım yeteneğine sahip İHA 'lar hakkında yeni sonuçlar sunmaktadır. Kanat ve kuyruk takımının uçak uzunlamasına ekseninde belli aralıklarda hareketlerinin, sabit hızda düz seviye uçuşunu varyans kısıtlı kontrolcüler ile sağlayan İHA'ların kontrol enerjisi üzerindeki etkileri bu konferans kâğıdının ana önceliğidir. Zanka-I adlı bilindik İHA kullanılmış ve onun datalarından ilgili analizlerde faydalanılmıştır. İlk olarak, sabit kanatlı İHA'ların dinamik modellemesi verilmiştir ve pasif başkalaşımın bu modellerdeki etkisi ayrıca araştırılmıştır. Sonrasında, pasif başkalaşımın çıkış varyansı kısıtlamalı kontrolcünün kontrol enerjisi üzerindeki etkisi ilgili İHA için 3 boyutlu Matlab grafiği kullanılarak sunulmuştur. Kontrol enerjisi ile kanat ve kuyruk takımının ileri geri hareketleri arasında kıyaslamalar yapılmışıtır.

Anahtar Kelimeler: Başkalaşım, IHA, OVC, Kontrol Enerjisi.

\section{Effect of Passive Morphing on UAVs Guided By Output Variance Constrained Controller}

\begin{abstract}
This paper presents novel results for UAVs having passive morphing property and guided by output variance constrained controller (i.e. OVC). Effects of forward and backward motions of wing and horizontal tail in aircraft longitudinal axis in prescribed interval on control energy of variance constrained controller satisfying straight level and constant speed flight of UAV are main interest of this conference paper. A known UAV named as ZANKA-I is used and its data is benefitted for related analyses. Firstly, dynamic modeling of fixed-wing UAVs is given and effect of passive morphing on these models is also investigated. Then, effect of passive morphing on control energy of output variance constrained controller for related UAV using 3-D Matlab graph is presented. Comparisons between control energy versus forward and backward motions of wing and horizontal tail are done.
\end{abstract}

Keywords: Morphing, UAV, OVC, Control Energy.

\footnotetext{
* Sorumlu Yazar: İskenderun Teknik Üniversitesi, Havacılık ve Uzay Bilimleri Fakültesi, Uçak Gövde-Motor Bölümü, Hatay, Türkiye, ORCID: 0000-0003-2412-4131, firat.sal@iste.edu.tr
} 


\section{Giriş}

Geçmiş 40-50 yılda insansız hava araçları (IHA'lar) gerek sivil gerekse de askeri amaçlar ile yaygın bir şekilde kullanılmıştır. İHA'ların böylesine yaygın bir şekilde kullanımının nedenleri şu şekilde sıralanabilir: Kolay imal-edilebilirlilik, ucuz işletme maliyeti, esnek yapısal donanım vb. Bu ve benzeri üstünlüklerinde dolayı İHA'lar birçok farklı uygulamalarda (ekin görüntülenmesi, ilaçlama, kıyı emniyet, vb.) kullanılmıştır. Referans [1]: Austin, 2010'da birçok farklı diğer uygulamaları görülüp incelenebilir. Bu makale çalışması kapsamında pasif başkalaşım özellikli, alçak irtifa, kısa menzil, kısa süre havada kalabilen bir İHA göz önünde bulundurulmuştur.

$\mathrm{Bu}$ makale çalışmasında literatürde bilinen birçok kontrolcüye (örneğin PID, LQR, LQG, Hinf) göre birçok üstünlük barındırmasından dolayı, çıkış varyansı kısıtlı kontrolcü, İngilizce bilinen adıyla Output Variance Constrained Controller (yani kısaltılmış hali OVC) kontrolcü uçuş kontrol sistemi olarak ilgili İHA'da seçilmiştir (bknz. Hsieh, Sketon ve Damra, 1989 [2] ve Zhu and Skelton, 1991 [3]). Pasif başkalaşım özellikli Zanka-I isimli bu İHA'nın otonom otomatik kontrolü OVC kontrolcüsü ile sağlanacaktır. OVC kontolcüsünün üstün özellklerden birisi ikinci derece bilgi kullanıyor olmasıdır. Bu kontrolcü aynı zamanda Kalman filtresi ile birlikte çalışmada ölçülemeyen durum değişkenleri olduğu durumuda bile sağlıklı kontrol sinyalleri üretebilmektedir.

Bu makale çalışmasında öncelikle pasif başkalaşım özellikli Zanka-I adlı İHA'nın dinamik modellemesi sunulmuştur. Peşinden Zanka-I adlı İHA'nın belli başlı fiziksel özellikleri listelenmiş̧ir. Sonrasında İHA'nın otonom uçuşunda kullanılacak OVC kontolcüsü özetlenmiştir. En sonda ise kanat ve kuyruk takımı pasif başkalaşımının kontrol enerjisine etkileri sunulmuştur. Bu çalışmada literatürde ilk defa pasik başkalaşım özellikli bir İHA üzerinde birçok üstün özelliği bulunan OVC kontrolcüsü ilk defa uygulanmış olunup, pasif başkalaşım parametreleri olan kanat ve kuyruk takımının enerji değerleir üzerinde etkileri analiz edilmiştir.

\section{Materyal ve Metot}

$\mathrm{Bu}$ bölümde kullanılan Zanka-I adlı pasif başkalaşım özellikli sabit kanatlı İHA'nın öncelikle dinamik modellenmesi ve sonrasında ise otonom kontrolünde kullanılacak OVC kontrolcüsü özetlenmiştir.

\subsection{Dinamik Modelleme}

Genel olarak sabit kanatlı İHA'ların modellenmesi içn şu referanslar incelenebilir: Nelson, 2007 [4] and Zagi, website [5]. Denklemler 1 ve 2' de sırası ile boylamasına ve yanlamasına durum-uzay modelleri sırası ile verilmiştir:

$$
\left[\begin{array}{c}
\Delta \dot{u} \\
\Delta \dot{w} \\
\Delta \dot{q} \\
\Delta \dot{\theta}
\end{array}\right]=\left[\begin{array}{cccc}
X_{u} & X_{w} & 0 & -g \\
Z_{u} & Z_{w} & u_{0} & 0 \\
M_{u}+M_{\dot{w}} Z_{w} & M_{w}+M_{\dot{w}} Z_{w} & M_{q}+M_{\dot{w}} u_{0} & 0 \\
0 & 0 & 1 & 0
\end{array}\right]\left[\begin{array}{c}
\Delta u \\
\Delta w \\
\Delta q \\
\Delta \theta
\end{array}\right]+\left[\begin{array}{cc}
X_{\delta_{T}} & X_{\delta_{e}} \\
Z_{\delta_{T}} & Z_{\delta_{e}} \\
M_{\delta_{T}}+M_{\dot{w}} Z_{\delta_{T}} & M_{\delta_{e}}+M_{\dot{w}} Z_{\delta_{e}} \\
0 & 0
\end{array}\right]\left[\begin{array}{c}
\Delta \delta_{T} \\
\Delta \delta_{e}
\end{array}\right]
$$

$$
\left[\begin{array}{c}
\Delta \dot{v} \\
\Delta \dot{p} \\
\Delta \dot{r} \\
\Delta \dot{\phi}
\end{array}\right]=\left[\begin{array}{cccc}
Y_{v} & Y_{p} & -\left(u_{0}-Y_{r}\right) & -g \cos \left(\theta_{0}\right) \\
L_{w}^{*}+\frac{I_{x z}}{I_{x}} N_{v}^{*} & L_{p}^{*}+\frac{I_{x z}}{I_{x}} N_{p}^{*} & L_{r}^{*}+\frac{I_{x z}}{I_{x}} N_{r}^{*} & 0 \\
N_{v}^{*}+\frac{I_{x z}}{I_{z}} L_{v}^{*} & N_{p}^{*}+\frac{I_{x z}}{I_{z}} L_{p}^{*} & N_{r}^{*}+\frac{I_{x z}}{I_{z}} L_{r}^{*} & 0 \\
0 & 1 & 0 & 0
\end{array}\right]\left[\begin{array}{c}
\Delta v \\
\Delta p \\
\Delta r \\
\Delta \phi
\end{array}\right]+\left[\begin{array}{cc}
0 & Y_{\delta_{r}} \\
L_{\delta_{a}}^{*}+\frac{I_{x z}}{I_{x}} N_{\delta_{a}}^{*} & L_{\delta_{r}}^{*}+\frac{I_{x z}}{I_{x}} N_{\delta_{r}}^{*} \\
N_{\delta_{a}}^{*}+\frac{I_{x z}}{I_{z}} L_{\delta_{a}}^{*} & N_{\delta_{r}}^{*}+\frac{I_{x z}}{I_{z}} L_{\delta_{r}}^{*} \\
0 & 0
\end{array}\right]\left[\begin{array}{c}
\Delta \delta_{a} \\
\Delta \delta_{r}
\end{array}\right]
$$

Şekil 1'de Zanka-I isimli pasif başkalaşım özellikli İHA'nın fotoğrafı paylaşılmıştır. Tablo 1'de ise bazı fiziksel özellikleri listelenmiştir. Ayrıca referanslar [7] ve [8]'de başkalaşım ayrınıtılı bir şekilde ele alınmıştır.

Tablo 1. Zanka-I’in Bazı Fiziksel Özellikleri (Oktay et al., 2016 [6])

\begin{tabular}{|c|c|}
\hline Nitelik & Miktar \\
\hline Toplam Ağırlık & $2.2 \mathrm{~kg}$ \\
\hline Boş A ğırlık & $2 \mathrm{~kg}$ \\
\hline Kanat Açıklı̆̆ & $1.3 \mathrm{~m}$ \\
\hline Kanat Kord Uzunluğu & $25 \mathrm{~cm}$ \\
\hline Kanat Açıklık Oranı & 5.2 \\
\hline Güç Sistemi & Brushless DC motor \\
\hline Maksimum Menzil & $30 \mathrm{~km}$ \\
\hline Maksimum Havada Kalıș Süresi & $0.5 \mathrm{~h}$ \\
\hline Maksimum HavadaKalış Süresi için Gerekli Hız & $40 \mathrm{~km} / \mathrm{h}$ \\
\hline
\end{tabular}



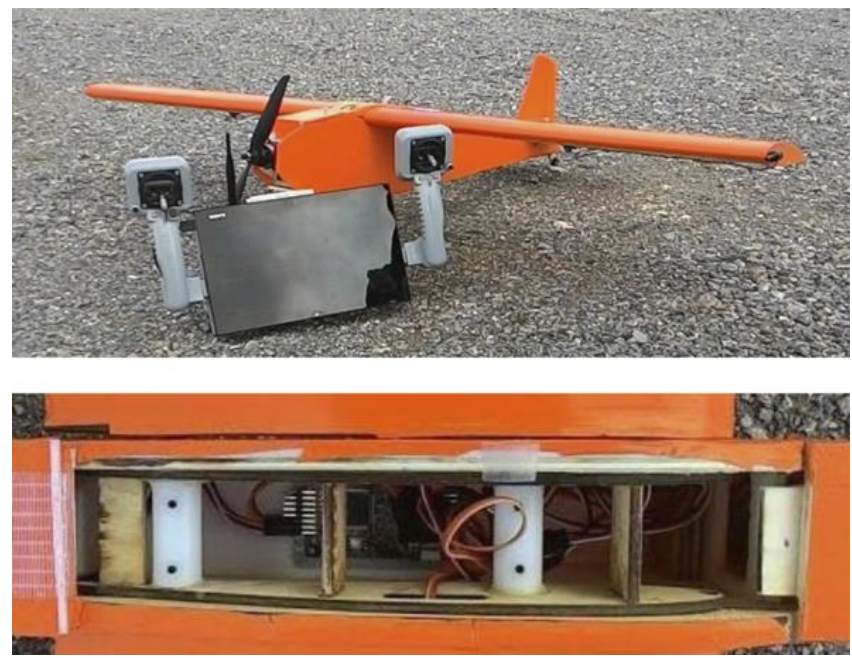

Şekil 1: Zanka-I'in Fotoğrafi (Oktay et al., 2016 [6])

\subsection{OVC Kontrolcüsü}

Pasif başkalaşım özellikli İHA'mızın kontrolünde speisifi bir kontrolcü olan OVC kontrolcüsünden faydalanılmıştır. Verilen sürekli, doğrusal, zamandan bağımsız, kararlı hale getirilebilir ve saptanabilir bir sistem için, $R>0$ pozitif tanımlı bir giriş penaltı matrisi mevcut iken, bir full dereceli dinamik kontrolcü aşağıdaki şartı sağladığında OVC kontrolcüsü oluşturulmuş olmaktadır.

$$
\min _{A_{C}, F, G} J=E_{\infty} u_{p}^{T} R u_{p}=\operatorname{tr}\left(R G X_{c_{j}} G^{T}\right)
$$

\section{Araştırma Sonuçları ve Tartışma}

Bu bölümde kanat ve kuyruk takımının hareketinin enerji değerine etkisi incelenmiş olunup, Şekil 2 deki gibi sonuçlar elde edilmiştir.

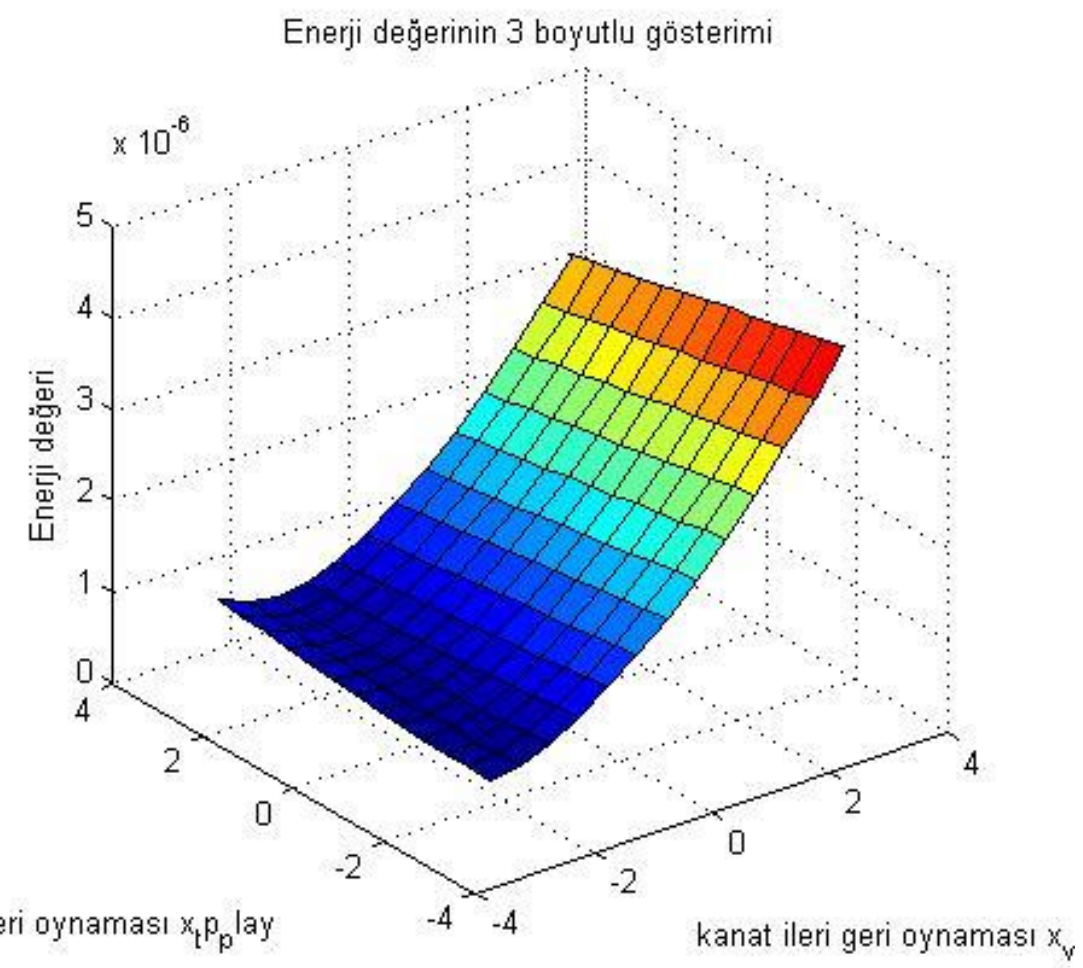

Şekil 2: Kanat Ve Kuyruk Takiminin Hareketinin Enerji Değerine Etkisi 
Şekil 2'de verilen 3 boyutlu Matlab çıktısı figürde, x- ekseni Zanka-I adlı İHA'nın kuyruk takımının (hem yatay kuyruk hem de düşey kuyruk birlikte eş zamanlı) ileri geri hareketini (+ ileri yönü, - geri yönü işaret etmekte) temsil etmekte, y- ekseni Zanka-I adlı İHA'nın kanadının ileri geri hareketini (+ ileri yönü, - geri yönü işaret etmekte) temsil etmekte, z- ekseni ise erişilen kuyruk takımı ve kanat konumuna göre uçuş kontrolünde kullanılan OVC kontolcüsünün enerji değerini ifade etmektedir. Bu şekilden anlaşılmaktadır ki kanat ileri hareketi enerji değerini arttırmakta, geri hareketi ise Zanka-I IHA konfigürasyonunda enerji değerini azaltmaktadır. Ayrıca kuyruk takımının ileri hareketi enerji değerini arttırmakta, geri hareketi ise Zanka-I İHA konfigürasyonunda enerji değerini azaltmaktadır. Pasif başkaşlaşım özellikli Zanka-I İHAsının üzerinde farklı kontrolcü çalılmaları için ayrıca Referanslar [9] ve [10]'da ele alınabilir.

\section{Sonuç}

$\mathrm{Bu}$ çalışma kapsamında çıkış varyansı kısıtlı kontrolcü (OVC) ile yönlendirilen pasif başkalaşım yeteneğine sahip İHA'lar hakkında yeni sonuçlar elde edilmiştir. Kanat ve kuyruk takımının uçak uzunlamasına ekseninde belli aralıklarda hareketlerinin, sabit hızda düz seviye uçuşunu varyans kısıtlı kontrolcüler ile sağlayan İHA'ların kontrol enerjisi üzerindeki etkileri gözlemlenmiştir. Kanat ileri hareketi enerji değerini arttırı iken, geri hareketi azaltmaktadır. Kuyruk takımı ileri hareketi de benzeri etkiyi yaratmaktadır.

\section{Teşekkür}

TÜBİTAK’a 114M856 no'lu proje üzerinden teşekkür ederiz.

\section{Kaynakça}

[1]Austin, R. (2010), Unmanned aircraft systems, Wiley.

[2] Hsieh, C., Skelton, R. E., and Damra, F. M. (1989), Minimum energy controllers with inequality constraints on output variances, Optimal Control Application and Methods, 10 (4), 347-366.

[3]Zhu, G. and Skelton, R. E. (1991), Mixed $L_{2}$ and $L_{\infty}$ problems by weight selection in quadratic optimal control. International Journal of Quadratic Optimal Control, 63 (5) : 1161-1176.

[4]Nelson, R. C. (2007), Flight Stability and Automatic Control. 2nd ed., McGraw-Hill, New York, chapters 2-6.

[5] Zagi-The original R/C EPP foam wing homepage (2015), http//:www.zagi.com.

[6]Oktay T., Konar M., Onay M. , Aydın M. , Mohamed M. A. (2016), Simultaneous small UAV and Autopilot System Design, Aircraft Engineering And Aerospace Technology, cilt.88, ss.818-834, 2016.

[7]Arik S., Turkmen I., Oktay T. (2018), Redesign of Morphing UAV for Simultaneous Improvement of Directional Stability and Maximum Lift/Drag Ratio, Advances In Electrical And Computer Engineering, cilt.18, ss.57-62.

[8]Oktay T., Çoban S. (2017), Simultaneous longitudinal and lateral flight control system design for both passive and active morphing TUAVs, Elektronika Ir Elektrotechnika, cilt.23, ss. 15-2017.

[9] Mohamed, M., Oktay, T., Konar, Autopilot Parameters Tunings For Best Performance, Simultaneous small UAV and autopilot system design, 8th Ankara International Aerospace Conference, Ankara, Türkiye, 10 - 12 Eylül 2015.

[10] Oktay, T., Konar, M., Onay, M., Mohamed, M., Integrated Autopilot Tunning and Unmanned Aerial Vehicle (UAV) Design, 6th European Conference For Aeronautics and Space Science, Krakow, Polonya, 29 June - 03 July 2015. 